\title{
Factores determinantes de fotosensibilidad y dermatosis más frecuentes
}

\section{More common determining factors for photosensitivity and dermatosis}

\author{
Katherine Dariana Rodríguez Lémus \\ Universidad de San Carlos de Guatemala \\ Keithlemus10@hotmail.com \\ https://orcid.org/0000-0001-8385-7939
}

Recibido: 29/06/2021

Aceptado: 01/09/2021

\section{Referencia del artículo}

Rodríguez Lémus, K. D. (2021). Factores determinantes de fotosensibilidad y dermatosis más frecuentes. Revista Diversidad Científica, 1(1). 177-183.

DOI: https://doi.org/10.36314/diversidad.v1i1.19

\section{Resumen}

En las enfermedades de la piel intervienen muchos factores fotosensibles y pueden llegar a causar lesiones agravantes en la epidermis, hasta causar riegos graves como el cáncer; y las altas tasas de exposición al sol, emiten una amplia cantidad de radiaciones electromagnéticas, pero las más importantes desde el punto de vista dermatológico, son las que pertenecen al espectro fotobiológico porque causan lesiones cutáneas (fotodermatosis). En general, parecen estar aumentando notablemente en los últimos años en todo el mundo, debido a la tendencia de las modificaciones ambientales provocadas por la contaminación, y ahora es uno de los principales o más consultado problema en salud pública en el mundo. Las organizaciones mundiales de la salud realizan investigaciones con el objetivo de obtener el índice UV más alto donde la ubicación geográfica es un importante determinante para la intensidad de los rayos UV, y su asociación con las enfermedades actínicas agudas de piel. Entre las dermatosis más comunes se mencionan: la urticaria solar, hydroa vacciniforme, erupción polimorfa lumínica, prurigo actínico, dermatitis actínica crónica, por lo cual se determina que la mayoría de pacientes con afecciones por dermatosis, se mantienen en constante exposición a la luz solar, provocando daño y alteración a la estructura de la epidermis, por lo que se puede encontrar una patología de tipo crónico, según el lapso de tiempo de exposición. 
Palabras clave: fotosensibilidad, ultravioleta, piel, lesiones por radiación, dermatosis

\begin{abstract}
Many photosensitive factors are involved in skin diseases and can lead to aggravating lesions in the epidermis, causing serious risks such as cancer; and the high rates of sun exposure, emit a large amount of electromagnetic radiation, but the most important from a dermatological point of view, are those that belong to the photobiological spectrum because they cause skin lesions (photodermatosis). Overall, they appear to be increasing markedly in recent years around the world, due to the trend of environmental changes caused by pollution, and is now one of the main or most consulted public health problem in the world. Global health organizations conduct research with the aim of achieving the highest UV index where geographic location is an important determinant for UV intensity, and their association with acute active skin diseases. Among the most common dermatosis are mentioned: solar hives, vacciniforme hydroa, polymorphic light rash, actinic prurigo, chronic actinic dermatitis, so it is determined that most patients with dermatosis conditions, are kept in constant exposure to sunlight, causing damage and alteration to the structure of the epidermis, so that a chronic pathology can be found, depending on the exposure time span.
\end{abstract}

Keywords: photosensitivity, UV, skin, radiation injuries, dermatosis 


\section{Introducción}

La presente investigación nos demuestra que las propiedades vigorizantes de la luz del sol han sido promovidas por los antiguos y por los modernos buscadores de la salud, pero las reacciones adversas producidas o evocadas por la energía ultravioleta que son muy diversas y su incidencia depende del cuadro clínico específico. En general, parecen estar aumentando notablemente en los últimos años en todo el mundo debido a la tendencia de las modificaciones ambientales provocadas por la contaminación.

Tiene el objetivo de descubrir e indagar sobre los riesgos de la exposición prolongada a los rayos del sol, ya que a consulta dermatológica se evidencian varios casos provocados por la radiación solar, teniendo efectos sobre la piel, los ojos y el sistema inmunológico. La luz solar es indispensable para la vida, una exposición excesiva puede ser en extremo peligrosa, conlleva riesgo como cánceres cutáneos y cataratas.

Las dermatosis son patologías que se presentan frecuentemente en poblaciones de países en vías de desarrollo. Sin embargo, hay pocos estudios en estos países que le den la importancia a estas enfermedades, las cuales suponen un gran problema de salud pública.

\section{Contenido \\ Prurigo actínico}

También conocida como enfermedad de Hutchinson, clínicamente se presenta con erupciones pápulo-nodulares, rash intenso, excoriaciones y que pueden dejar pigmentaciones o cicatrices en las áreas fotoexpuestas; se pueden extender hacia sitios no expuestos (espalda y glúteos) (Zeas 2003, citado en Auquilla y Quizhpe 2016).

\section{Hydroa vacciniforme}

La condición se ve igual en ambos sexos. Tiene un curso intermitente, con aparición de erupciones en placas y vesículas en las áreas expuestas a la luz, especialmente en el rostro, brazos y manos, pocas horas después de la exposición al sol. Después de 4 o 6 semanas aparecen cicatrices varioliformes. No hay exámenes de labora- 
torio de diagnóstico de la enfermedad y el diagnóstico se realiza principalmente por motivos clínicos, apoyado por la histología muy característica y pruebas de fotosensibilidad (Cobán, et al. 2011).

\section{Erupción polimorfa lumínica}

Enfermedad muy común, afecta sobre todo a mujeres jóvenes en zonas tropicales. La prevalencia en la población general varía se estima en $10-20 \%$. Tiene un curso intermitente relacionado con las estaciones (primavera y verano). El cuadro clínico se caracteriza por erupciones papulovesiculares en áreas expuestas, eritema, picor que se resuelven sin dejar cicatriz, parece en horas tras la exposición solar y se resuelve en días. Se debe hacer diagnóstico diferencial con Lupus eritematoso sistémico (Segurado, et al. 2002).

\section{Urticaria solar}

Es una enfermedad poco frecuente, mediada por una reacción antígeno-anticuerpo, se presenta pocos minutos después de la exposición solar, con aparición de quemazón seguido de eritema, prurito y habones. Afecta a todas las edades y sin diferencia entre hombres y mujeres. Los rayos UVA son los que tienen mayor importancia en esta patología, la gravedad de las lesiones depende de la intensidad y tiempo de exposición. El diagnóstico se confirma con una fotoprueba y el tratamiento se basa en fototerapia de desensibilización (Raigosa, et al. 2017).

\section{Dermatitis actínica crónica}

Enfermedad rara, afecta a hombres mayores de 50 años. Se han postulado dos teorías: fotoalérgica y fototóxica. La primera teoría se apoya en la relación con alérgenos tales como fragancias, cauchos, plantas, fármacos. La segunda teoría se basa en estudios que establecen un daño directo en el ADN. El cuadro clínico se caracteriza por lesiones excoriativas más prurito, erupciones eczematosas que pueden liquenificarse. Las lesiones pueden extenderse a zonas no expuestas al sol e incluso provocar eritrodermia (pseudolinfoma). El tratamiento se basa en la fotoprotección, uso de corticoides, PUVA-terapia e inmunosupresores (Acosta, 2015). 


\section{Conclusión}

Las lesiones fotosensibles más comunes como prurigo actínico, urticaria solar, hydroa vacciniforme, erupción polimorfa lumínica, dermatitis actínica crónica las cuales se distinguen por sus características clínicas y que por sus factores de riesgo en común pueden predisponer afecciones crónicas o riesgo cancerígeno. Las enfermedades de la piel varían según el área geográfica, factores ambientales y sus características sociodemográficas como: la latitud, nubosidad, altura del sol, reflexión, capa de ozono que influyen determinadamente en la modificación en la piel; provocando una quemadura solar y su constante exposición puede llegar a la transformación de células cancerígenas.

\section{Declaración}

El estudio se realizó con fines médicos, de acuerdo a las directrices del Código de Ética y Buenas Prácticas de Publicaciones COPE.

\section{Conflicto de intereses}

La autora declara no tener ningún conflicto de intereses.

\section{Referencias}

Acosta, A., Nova, J., Sánchez, G., Rodríguez, A., Rueda, X., Valbuena, M., Cepeda, M., Ramirez, A. F., Jimenez, G., Herrera, H., Arévalo, I., Segura, O. (2015). Guía de atención integral con evaluación económica para la prevención, el diagnóstico, el tratamiento y el seguimiento del cáncer de piel no melanoma: carcinoma basocelular (en línea). Revista de la Asociación Colombiana de Dermatología y Cirugía Dermatológica 23(4):258-296. DOI: https://doi.org/10.29176/2590843X.282

Arenas Guzmán, R. (2013). La piel (libro electrónico). In Dermatología: atlas, diagnóstico y tratamiento. 5 ed. México, McGraw-Hill. p. 1-24.

Çoban, M., Kocabas, E., Temiz, P., Ertan, P. y Ermertcan, A. T. (2011). Papulovesicular eruption located on the face and extremities in a child (en línea). In- 
dian Journal of Dermatoly, Venereol and Leprology 77(5):627-628. DOI: $10.4103 / 0378-6323.84088$

López Villaescusa, M. T., Robuschi Lestouquet, F., Negrín González, J., Muñoz González, R. C., Landa García, R., Conde-Salazar, L. (2012). Dermatitis clínica crónica en el mundo laboral (en línea). Revista Medicina y Seguridad del Trabajo 58(227):128-135. DOI: https://dx.doi.org/10.4321/S0465-546X2012000200006

Raigosa, M., Toro, Y. y Sánchez, J. 2017. Urticaria solar: reporte de un caso y revisión de la literatura (en línea). Revista Alergia México 64(3):371-375. DOI: https:// dx.doi.org/ 10.29262/ram.v64i3.202

Segurado Rodríguez, M. A., Guerra Tapia, A. y Iglesias Díez, L. (2002). Hydroa Vacciniforme: una rara forma de fotosensibilidad infantil (en línea). Revista Actas Dermo-Sifiliográficas 93(7):464-466. Disponible en https://actasdermo.org/ es-hydroa-vacciniforme-una-rara-forma- articulo-13036371

Zeas D, I. M., Ordóñez V. M. (2016). Dermatología básica para el médico general (en línea). Cuenca, Ecuador, Universidad de Cuenca. 140 p. Disponible en https:// dspace.ucuenca.edu.ec/bitstream/123456789/26151/3/DERMATOLOGI\%20 BASICA.pdf

\section{Sobre la autora \\ Katherine Dariana Rodríguez Lémus}

Es Médica y Cirujana egresada de la Universidad de San Carlos de Guatemala con especialización en administración y mantenimiento hospitalario de la Escuela de estudios de Postgrados USAC. Ha realizado investigaciones sobre caracterización del aborto en edades riesgo, propuesta de un sistema de señalización hospitalaria con el fin de facilitar la identificación y señalización de ambientes y flujos de circulación, Hospital general San Juan de Dios, área de emergencia, zona 1, Guatemala, factores determinantes de fotosensibilidad y dermatosis más frecuentes. 
Copyright (c) Katherine Dariana Rodríguez Lémus

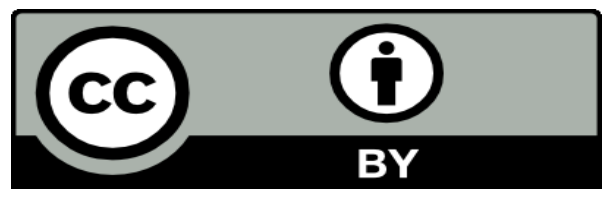

Este texto está protegido por una licencia CreativeCommons 4.0.

Usted es libre para compartir, copiar y redistribuir el material en cualquier medio o formato y adaptar el documento, remezclar, transformar y crear a partir del material para cualquier propósito, incluso comercialmente, siempre que cumpla la condición de atribución: usted debe reconocer el crédito de una obra de manera adecuada, proporcionar un enlace a la licencia, e indicar si se han realizado cambios. Puede hacerlo en cualquier forma razonable, pero no de forma tal que sugiera que tiene el apoyo del licenciante o lo recibe por el uso que hace. 